\title{
The sweet smell of success: Optimizing viability assessment protocols
}

\author{
Raymond Russell, MD, PhD, MASNC \\ a Division of Cardiology, Department of Medicine, Alpert Medical School of Brown University, \\ Providence, RI
}

Received Jul 7, 2020; accepted Jul 7, 2020

doi: $10.1007 / \mathrm{s} 12350-020-02282-2$

\section{See related article, pp. 248-250}

The concept of assessing myocardial viability with metabolic imaging is easy to comprehend. In contrast, the actual performance of the study can be much more challenging. Identifying viable myocardium with PET imaging of F-18-fluorodeoxyglucose (FDG) is based on the existential concept that viable cardiac myocytes possess the metabolic machinery to produce energy in the form of ATP. That ATP is used to phosphorylate glucose (as well as FDG) in the first, committed step of glycolysis. Phosphorylation of FDG traps the radiotracer in viable cells. To optimize the signal provided by FDG, it is important to maximize the amount of FDG that is transported into the myocyte. It is at this point that imaging of myocardial viability with FDG can become laborious and challenging.

Myocardial glucose uptake is regulated by the facilitative glucose transporters, GLUT1 and GLUT4. While GLUT1 is responsible for basal uptake of glucose by cardiac myocytes, GLUT4 is recruited to the surface of cardiac myocytes by insulin stimulation of insulin receptors and signaling through the PI-3K/Akt pathway. ${ }^{1}$ In addition, GLUT4 can be recruited to the cell surface by ischemia through the AMP-activated protein kinase pathway. ${ }^{1}$ Because these two pathways operate independently, it is possible to observe additive effects of insulin and ischemia on myocardial glucose uptake. ${ }^{2}$ Therefore, to maximize FDG uptake for a PET viability

Reprint requests: Raymond Russell, MD, PhD, MASNC, Division of Cardiology, Department of Medicine, Alpert Medical School of Brown University, 593 Eddy Street, APC737, Providence, RI 02903; raymond_russell@brown.edu

J Nucl Cardiol 2022;29:248-50.

$1071-3581 / \$ 34.00$

Copyright (C) 2020 American Society of Nuclear Cardiology. study, clinicians utilize insulin stimulation to increase the uptake of the radiotracer. The vast majority of imaging protocols rely on stimulating insulin release through the ingestion of glucose in nondiabetic patients, and, depending on the degree of hyperglycemia in a diabetic patient, either the ingestion of a lower amount of glucose or through insulin injection. The American Society of Nuclear Cardiology (ASNC) provides a useful protocol for the metabolic preparation for viability studies that is used in most nuclear cardiology labs ${ }^{3}$ (Table 1). There are other protocols that utilize intravenous infusion of glucose or a glucose/insulin clamp to increase myocardial glucose uptake. These protocols can be labor-intensive and time-consuming. In addition, acipimox can be used outside the United States to decrease serum triglyceride concentrations and thereby increase myocardial reliance on glucose. ${ }^{4}$ However, because of its simplicity, the oral glucose load is most widely used method to optimize FDG-PET images for viability assessment.

When comparing different protocols for viability assessment, or for that matter any study that we perform in nuclear cardiology, it is important to consider several factors. First, and most importantly, the safety of the procedure. For viability assessment, the safety is related to the avoidance of hypoglycemia. Second, the quality of the images, namely the myocardial uptake of FDG compared to surrounding tissues. Finally, the amount of time required to perform the procedure. As can be determined by the ASNC guidelines, the minimum time that is required for glucose loading and achieving a goal blood glucose level of $100-140 \mathrm{mg} / \mathrm{dL}$ (5.55$7.77 \mathrm{mmol} / \mathrm{L}$ ) is 45 minutes. Practically, however, it often takes more than 45 minutes depending on the rate of decline in the blood glucose level after the oral glucose load. This, in turn, is dependent on the degree of insulin sensitivity in the individual patients.

In this issue of the Journal, Chen et al. ${ }^{5}$ evaluated the efficiency and safety of a metabolic preparation 
Table 1. Summary of guidelines from the American Society of Nuclear Cardiology for the metabolic preparation for a PET viability study

\section{Procedure}

Fasting period

Oral glucose load

Blood glucose maintenance

\section{Radiotracer administration}

\section{Steps for standardization}

\author{
Step 1: Fast patient 6-12 h (preferred), $<6 \mathrm{~h}$ (optional)
}

Step 2: Check blood glucose (BG) and then glucose load

If fasting $B G<250 \mathrm{mg} / \mathrm{dL}(13.9 \mathrm{mmol} / \mathrm{L})$, then:

(1) Oral glucose load (25-100 g)

(2) Monitor BG 45-60 min after administration

If fasting $B G>250 \mathrm{mg} / \mathrm{dL}(13.9 \mathrm{mmol} / \mathrm{L})$, then refer to Blood glucose maintenance (below)

After 45-60 min:

If $B G$ is $130-140 \mathrm{mg} / \mathrm{dL}(7.22-7.78 \mathrm{mmol} / \mathrm{L})$, then give $1 \mathrm{U}$ regular insulin, i.v If $B G$ is $140-160 \mathrm{mg} / \mathrm{dL}(7.78-8.89 \mathrm{mmol} / \mathrm{L})$, then give $2 \mathrm{U}$ regular insulin, i.v If $B G$ is $160-180 \mathrm{mg} / \mathrm{dL}(8.89-10 \mathrm{mmol} / \mathrm{L})$, then give $3 \mathrm{U}$ regular insulin, i.v If $B G$ is $180-200 \mathrm{mg} / \mathrm{dL}(10-11.11 \mathrm{mmol} / \mathrm{L})$, then give $5 \mathrm{U}$ regular insulin, i.v If $B G$ is $>200 \mathrm{mg} / \mathrm{dL}$ ( $>11.11 \mathrm{mmol} / \mathrm{L}$ ), then notify physician

Inject FDG when $B G$ is $100-140 \mathrm{mg} / \mathrm{dL}(5.55-7.77 \mathrm{mmol} / \mathrm{L})$

Adapted from Ref. $^{3}$

based on an insulin loading protocol compared to a standardized glucose loading protocol for FDG-PET imaging. In this study, patients undergoing an FDG-PET viability study were randomized to receive either 2-10 U of intravenous insulin based on the patient's fasting blood glucose or a graded amount of glucose (between 10-20 and 40-50 g) based on their fasting blood glucose. In addition, diabetic patients in the insulin loading arm of the study were given an additional $10 \%$ more insulin. In the insulin loading group, blood glucose was measured every 5-10 minutes compared to after 4560 minutes in the glucose load group, increasing the amount of effort required by staff and discomfort to the patient. Additional insulin was given as needed in both groups to bring the blood glucose down to the target value of $100-140 \mathrm{mg} / \mathrm{dL} \quad(5.55-7.77 \mathrm{mmol} / \mathrm{L})$. The quality of the cardiac images was assessed by comparing myocardial FDG uptake to liver FDG uptake. In addition, the time required to perform the protocol and the development of hypoglycemia were also compared between the two groups.

The quality of the images was considered acceptable in all of the insulin loading studies but in only 25 of 30 glucose loading studies. This was based on a myocardium/liver ratio of FDG uptake $>1$. All of the patients in the glucose loading studies with unacceptable studies were either overweight or obese, although the mean weights and BMI values for the two groups were not statistically different. It is difficult to determine if the issue with the quality of the images in the glucose loading group was attributable specifically to the preparation or if the body habitus of the patients contributed significantly to the findings. With respect to other characteristics of the images, the standardized uptake values (SUV) for the myocardium were similar in the two groups, as were the SUV values for the liver. Interestingly, the SUV values for skeletal muscle (another insulin-sensitive tissue) were actually lower in the insulin loading group. It is not clear why there would be less glucose uptake in skeletal muscle in the insulin loading group when the myocardial uptake was similar in both groups.

With respect to the safety of the insulin loading protocol, there were fewer episodes of hypoglycemia in the insulin loading group (2/30) compared to the glucose loading group (6/30). However, it is important to note that hypoglycemia was defined by the onset of symptoms typical for hypoglycemia and that only 4 of the 8 patients experiencing hypoglycemic symptoms had a final blood glucose value below $100 \mathrm{mg} / \mathrm{dL}$ (5.55 mmol/ $\mathrm{L})$. It is possible that the lower incidence of hypoglycemia in the insulin loading group was due to the more frequent monitoring of blood glucose levels during the protocol, as both groups ultimately received the same mean dose of insulin to achieve target blood glucose levels.

With respect to the amount of time required between the start of the loading procedure and the achievement of the target blood glucose levels, use of the insulin loading protocol reduced the time by 71 minutes to a mean value of 24 minutes. Diabetic patients receiving the insulin load required on average 7 
more minutes before they achieved a target blood glucose level compared to the nondiabetic individuals. This time is certainly less than the estimated minimum of 45 minutes based on the ASNC guidelines and also shorter than a reported 39-minute time obtained with a glucose/insulin/potassium infusion. ${ }^{6}$ It is difficult to know what impact more frequent blood glucose monitoring had on the observed differences in time to FDG injection since blood glucose levels were assessed every 5-10 minutes in the insulin loading group, after 4560 minutes in the glucose loading group, and every 15 minutes in the glucose/insulin/potassium infusion study. ${ }^{6}$

While the study by Chen et al. suggests that obtaining the necessary metabolic conditions to acquire interpretable FDG-PET viability images safely in a shorter period of time is indeed feasible, it does come at the cost of more frequent monitoring of blood glucose levels. In addition, the study did not determine the contribution of body habitus on image quality, nor did it evaluate the impact of the different protocols on medical decision making and ultimate patient outcome. Further studies of this protocol in larger groups of individuals will be required to determine just how sweet it is.

\section{References}

1. Young LH, Coven DL, Russell RR. Cellular and molecular regulation of cardiac glucose transport. J Nucl Cardiol 2000;7:267-76

2. Russell RR III, Yin R, Caplan MJ, Hu X, Ren J, Shulman GI, et al. Additive effects of hyperinsulinemia and ischemia on myocardial GLUT1 and GLUT4 translocation in vivo. Circulation 1998;98:2180-6.

3. Dilsizian V, Bacharach SL, Beanlands RS, Bergmann SR, Delbeke D, Dorbala S, et al. ASNC imaging guidelines/SNMMI procedure standard for positron emission tomography (PET) nuclear cardiology procedures. J Nucl Cardiol 2016;23:1187-226.

4. Nuutila P, Knuuti MJ, Raitakari M, Ruotsalainen ULLA, Teras M, Voipio-Pulkki LM, et al. Effect of antilipolysis on heart and skeletal muscle glucose uptake in overnight fasted humans. Am J Physiol 1994;267:E941-6.

5. Chen YC, Wang QQ, Wang YH, Zhuo HL, Dai RZ. Intravenous regular insulin is an efficient and safe procedure for obtaining highquality cardiac 18F-FDG PET images: An open-label, single-center, randomized controlled prospective trial. J Nucl Cardiol 2020.

6. Martin WH, Jones RC, Delbeke D, Sandler MP. A simplified intravenous glucose loading protocol for fluorine-18 fluorodeoxyglucose cardiac single-photon emission tomography. Eur $\mathbf{J}$ Nucl Med 1997;24:1291-7.

Publisher's Note Springer Nature remains neutral with regard to jurisdictional claims in published maps and institutional affiliations. 